\title{
Relationships Between Perception toward Assessment with Learning Result of Student
}

\author{
Muchlis \\ Chemistry Department, FMIPA \\ Universitas Negeri Surabaya \\ muchlis@unesa.ac.id \\ Subandi \\ Prof., Graduate School, \\ Universitas Negeri Malang \\ Subandi.fmipa@um.ac.id
}

\author{
Suhadi Ibnu \\ Prof., Graduate School, \\ Universitas Negeri Malang \\ suhadi.ibnu.fmipa@um.ac.id \\ Siti Marfuah \\ Graduate School, \\ Universitas Negeri Malang \\ Siti.marfuah.fmipa@um.ac.id
}

\begin{abstract}
This research aim to describe relationships between perception toward assessment with learning result of student. Perception toward assessment it means student percepts assessment as assessment of learning (AoL), assessment for learning (AfL) or assessment as learning (AaL). Subject research is 35 students of Chemistry Education of Surabaya State University. Research design is one shot case study repeated because one student group given three times different treatment without control group. Relationships between both is analyzed by chi-square test and percentage of student amount that obtaining the highest and lower in learning result appropriate its perception toward assessment. Result of analysis indicate that between perception toward assessment with learning result of student are not significant in relationships. However, if student's the highest and lower categories in learning result are added at implementation of AoL, AfL and AaL are always predominated by student which percept assessment successively as AoL, AfL and AaL. Amount of student which is obtaining the highest and lower in learning result at implementation of $\mathrm{AoL}$ is 15 (42,9\%), $14(40 \%)$ and $2(5,7 \%)$ student which percept assessment successively as AoL, AfL and AaL. Amount of student which is obtaining the highest and lower in result learning at implementation of $A f L$ is $23(65,7 \%)$, $6(17,1 \%)$ and $0(0 \%)$ student which is percept assessment successively as AoL, AfL and AaL. Amount of student which is obtaining the highest and lower in result learning at implementation of $\mathrm{AaL}$ is 20 ( $57,1 \%), 6(17,1 \%)$ and $3(8,6 \%)$ student which is percept assessment successively as AoL, AfL and AaL.
\end{abstract}

Keywords-perception toward assessment, learning result of student, assessment of learning, assessment for learning, assessment as learning.

\section{INTRODUCTION}

Assessment of learning is also called assessment for summative purposes or summative assessment [1]. Assessment of learning is a process collecting and interpreting of evidence for learning summary at periodic time, to make decision of student learning quality based on criteria and mark of value that represented of quality. The information gathered is used by the teacher to summarize learning at a given point in time, and this summary is used to make judgements about the quality of student learning on the basis of established criteria, to assign a value to represent that quality, and to support the communication of information about achievement to students themselves, parents, teachers, and others.

Assessment for learning focus on assessment for formative that procedure at formal and informal range that done by teacher as part of teaching-learning normal process and information use teacher and student to modified and to improve learning and understanding [2]. Assessment for learning offering alternative perspective towards assessment in school, or the simple words assessment for learning shift from summative assessment to formative assessment, from decision taking to create description that it used to decide next step in a students' learning.

Not Comparing student with the others at implementation AaL. Assessment as learning make teacher change knowledge bearers to knowledge guides where the teacher guides students by to understands "their cognitive process" so students learn monitoring their understanding and make adjustments. Assessment as learning is students reflect their work and make them great role towards their work. According to Earl and Katz (2006) that in assessment as learning, the mind of students about their learning, strategies to support and to advance their learning, and mechanism students to make adjustments and help their learning.

The idea that assessment can help students in learning is not new, but assessment for learning is a one of the most powerful ways of improving student achievement [3]. Similarly, according to [4] that student achievement can be improved through assessment for learning. Other research results also showed that the application of consistent assessment for learning will produce something extraordinary, if not something that has never happened, at least student achievement [5]. Report of OECD (2005) show assessment for learning (formative assessment) increase aim of life long education, level of learning result, extend of learning outcome and learn to learning skill. It so happen [6] report that the use of a wiki to emphasize the application of assessment as learning has enriched student learning experiences that can improve professional 
student outcomes in Tertiary Educations. This evidences gives prospect that the highest student's perception toward assessment so the highest it's learning result.

Perception is 1) ability see, hear, or become aware of something through the sense; 2) the way in which something

is regarded, understood or interpreted. Perception towards assessment is defined as student behavior recognize assessment in learning research moment conducted.

Many researchers have studied perception toward assessment with learning result. But assessment in here it means assessment types, assessment tasks dan classroom assessment environment. While, study relationships between perception toward assessment with learning result is not reported. Examples, report that there is weak correlation between perceptions of assessment type (oral test, written test, alternatives test) with assessment score. Alkharusi (2013) report that albeit not correlation causality between perceptions of assessment task (congruence with planned learning, authenticity, student consultation, transparancy, diversity) with motivation and learning, but this result study can help to understand how do silent aspects of assessment task related meaningfull towards motivation and learning [7]. The same way, research of Cheng, $\mathrm{Wu}$, and Liu (2015) that study correlation between perception of assessment task and classroom assessment environment (learning-oriented assessment environment, performance-oriented assessment environment) [8]. The result study Cheng, $\mathrm{Wu}$, and Liu (2015) show that congruence with planned learning and student consultation are positive predictor of learning-oriented assessment environment and negative predictor of performanceoriented assessment environment [8]. Whiles no reports of relationships between perception towards assessment (it's means assessment of learning, assessment for learning, and assessment as learning) with learning result.

\section{METHOD}

Research design use one shot case study repeated because one group give 3 times treatment without class control. Research subject is 35 students of Chemistry Education Program at Mathematics and Science Faculty in Surabaya State University. Three treatment in here are implementation AoL, AfL and AaL in a series at meeting 1-4, 6-9, and 11-14. Measuring of the perception toward assessment and learning result is done at meeting 5, 10 and 15. The learning material and research instrument have validated and test tried. The measuring result of perception toward assessment and learning result are tested chi-square test and percentage of student amount that the highest, lower and the lowest in learning result achievement in a row of it's perception toward assessment at each treatment.

\section{RESULTS OF RESEARCH AND DISCUSSION}

Student percept assessment as AoL, AfL and AaL is given score 1, 2 and 3 respectively. Score recapitulation of perception toward assessment written in Table 1. There are $8,6 \% ; 42,9 \%$ and $48,5 \%$ student percept assessment as AoL, AfL and AaL respectively at implementation AoL.
There are $0 \% ; 20 \%$ and $80 \%$ student percept assessment as AoL, AfL and AaL respectively at implementation AfL. There are $8,6 \% ; 20 \%$ and $71,4 \%$ student percept assessment as AoL, AfL and $\mathrm{AaL}$ respectively at implementation AaL.

TABLE I. SCORE PERCEPTION TOWARD ASSESSMENT AT IMPLEMENTATION OF AOL,AFL AND AAL

\begin{tabular}{|c|c|c|c|c|c|c|c|c|c|}
\hline \multirow[t]{2}{*}{ No. } & \multirow[t]{2}{*}{$\begin{array}{l}\text { Studen } \\
\text { t Name }\end{array}$} & \multicolumn{3}{|c|}{$\begin{array}{c}\text { Score } \\
\text { perception } \\
\text { toward } \\
\text { assessment } \\
\text { at } \\
\text { implementa } \\
\text { tion of }\end{array}$} & \multirow[t]{2}{*}{ No. } & \multirow[t]{2}{*}{$\begin{array}{l}\text { Studen } \\
\text { t Name }\end{array}$} & \multicolumn{3}{|c|}{$\begin{array}{c}\text { Score } \\
\text { perception } \\
\text { toward } \\
\text { assessmen } \\
\text { t at } \\
\text { implement } \\
\text { ation of }\end{array}$} \\
\hline & & \begin{tabular}{l|l}
$A$ \\
$o$ \\
$L$
\end{tabular} & $\begin{array}{c}A f \\
L\end{array}$ & $\begin{array}{c}A a \\
L\end{array}$ & & & & & $\begin{array}{c}A a \\
L\end{array}$ \\
\hline 1 & PIKM & 2 & 3 & 2 & 19 & ST & 3 & 3 & 3 \\
\hline 2 & RTA & 3 & 3 & 3 & 20 & $\mathrm{~S}$ & 2 & 2 & 3 \\
\hline 3 & MFSK & 3 & 3 & 3 & 21 & RKN & 2 & 2 & 3 \\
\hline 4 & MSR & 2 & 3 & 3 & 22 & AFN & 2 & 2 & 3 \\
\hline 5 & RED & 3 & 2 & 1 & 23 & EAP & 2 & 3 & 3 \\
\hline 6 & BBM & 2 & 3 & 3 & 24 & $\mathrm{RA}$ & 3 & 3 & 3 \\
\hline 7 & FRS & 3 & 3 & 3 & 25 & CYS & 2 & 3 & 3 \\
\hline 8 & MDAR & 1 & 3 & 2 & 26 & UNH & 2 & 2 & 2 \\
\hline 9 & FA & 3 & 3 & 3 & 27 & LSP & 3 & 3 & 3 \\
\hline 10 & BSYS & 2 & 3 & 2 & 28 & $\mathrm{AR}$ & 3 & 2 & 3 \\
\hline 11 & KTR & 2 & 3 & 3 & 29 & DW & 3 & 3 & 3 \\
\hline 12 & $\mathrm{ZS}$ & 3 & 3 & 3 & 30 & RAJ & 3 & 2 & 2 \\
\hline 13 & HW & 1 & 3 & 2 & 31 & AWS & 3 & 3 & 1 \\
\hline 14 & VAS & 2 & 3 & 3 & 32 & SNAA & 3 & 3 & 3 \\
\hline 15 & SPA & 3 & 3 & 3 & 33 & ARF & 3 & 3 & 3 \\
\hline 16 & MM & 2 & 3 & 3 & 34 & BEP & 2 & 3 & 3 \\
\hline 17 & MFR & 1 & 3 & 1 & 35 & FDN & 3 & 3 & 2 \\
\hline 18 & RK & 2 & 3 & 3 & & & & & \\
\hline
\end{tabular}
AaL is shown in Table 2. Table 3 show the result learning of student is classified in the lowest ( $\mathrm{X}<\mathrm{M}-1 \mathrm{SD})$, lower (M-1SD $\leq \mathrm{X}<\mathrm{M}+1 \mathrm{SD})$, and the highest categories $(\mathrm{X} \geq$ $\mathrm{M}+1 \mathrm{SD})$ (Azwar, 2012).

TABLE II LEARNING RESULT AT IMPLEMENTATION OF AOL,

$$
\text { AFL AND AAL }
$$

\begin{tabular}{|c|c|c|c|c|c|c|c|c|c|}
\hline \multirow[t]{2}{*}{ No. } & \multirow{2}{*}{$\begin{array}{c}\text { Stude } \\
\text { nt } \\
\text { Name }\end{array}$} & \multicolumn{3}{|c|}{$\begin{array}{c}\text { Learning } \\
\text { result at } \\
\text { implementat } \\
\text { ion of }\end{array}$} & \multirow[t]{2}{*}{ No. } & \multirow{2}{*}{$\begin{array}{c}\text { Stude } \\
\text { nt } \\
\text { Name }\end{array}$} & \multicolumn{3}{|c|}{$\begin{array}{c}\text { Learning } \\
\text { result at } \\
\text { implementat } \\
\text { ion of }\end{array}$} \\
\hline & & $\begin{array}{l}A \\
o \\
L\end{array}$ & $\begin{array}{c}A f \\
L\end{array}$ & $\begin{array}{l}A \\
a \\
L\end{array}$ & & & $\begin{array}{l}A \\
o \\
L\end{array}$ & $\begin{array}{l}\text { A } \\
\text { fL }\end{array}$ & $\begin{array}{l}\text { A } \\
\mathrm{a} \\
\mathrm{L}\end{array}$ \\
\hline 1 & $\begin{array}{c}\text { PIK } \\
\text { M }\end{array}$ & $\begin{array}{l}45 \\
.8\end{array}$ & $\begin{array}{r}86 \\
.4\end{array}$ & $\begin{array}{r}91 \\
.7\end{array}$ & 19 & ST & $\begin{array}{l}66 \\
.7\end{array}$ & $\begin{array}{l}90 \\
.9\end{array}$ & $\begin{array}{r}83 \\
.3 \\
\end{array}$ \\
\hline 2 & RTA & $\begin{array}{r}37 \\
.5\end{array}$ & $\begin{array}{l}72 \\
.7\end{array}$ & $\begin{array}{l}75 \\
.0\end{array}$ & 20 & $\mathrm{~S}$ & $\begin{array}{l}45 \\
.8\end{array}$ & $\begin{array}{c}81 \\
.8\end{array}$ & $\begin{array}{r}83 \\
.3\end{array}$ \\
\hline 3 & $\begin{array}{c}\text { MFS } \\
\text { K }\end{array}$ & $\begin{array}{l}37 \\
.5\end{array}$ & $\begin{array}{l}86 \\
.4\end{array}$ & $\begin{array}{l}87 \\
.5\end{array}$ & 21 & RKN & $\begin{array}{c}45 \\
.8\end{array}$ & $\begin{array}{r}95 \\
.5\end{array}$ & $\begin{array}{l}91 \\
.7\end{array}$ \\
\hline 4 & MSR & 50 & 50 & 58 & 22 & AFN & 58 & 68 & 91 \\
\hline
\end{tabular}




\begin{tabular}{|c|c|c|c|c|c|c|c|c|c|}
\hline \multirow{3}{*}{ No. } & \multirow{2}{*}{$\begin{array}{c}\text { Stude } \\
\text { nt } \\
\text { Name }\end{array}$} & \multicolumn{3}{|c|}{$\begin{array}{c}\text { Learning } \\
\text { result at } \\
\text { implementat } \\
\text { ion of }\end{array}$} & \multirow[t]{2}{*}{ No. } & \multirow{2}{*}{$\begin{array}{c}\text { Stude } \\
\text { nt } \\
\text { Name }\end{array}$} & \multicolumn{3}{|c|}{$\begin{array}{c}\text { Learning } \\
\text { result at } \\
\text { implementat } \\
\text { ion of }\end{array}$} \\
\hline & & $\begin{array}{l}A \\
o \\
L\end{array}$ & $\begin{array}{c}A f \\
L\end{array}$ & $\begin{array}{c}A \\
a \\
L\end{array}$ & & & $\begin{array}{c}A \\
o \\
L\end{array}$ & $\begin{array}{c}\text { A } \\
\mathrm{fL}\end{array}$ & $\begin{array}{c}\mathrm{A} \\
\mathrm{a} \\
\mathrm{L}\end{array}$ \\
\hline & & .0 & .0 & .3 & & & .3 & .2 & .7 \\
\hline 5 & RED & $\begin{array}{l}62 \\
.5\end{array}$ & $\begin{array}{c}10 \\
0\end{array}$ & $\begin{array}{l}87 \\
.5\end{array}$ & 23 & EAP & $\begin{array}{c}58 \\
.3\end{array}$ & $\begin{array}{l}77 \\
.3\end{array}$ & $\begin{array}{c}66 \\
.7\end{array}$ \\
\hline 6 & BBM & $\begin{array}{r}41 \\
.7 \\
\end{array}$ & $\begin{array}{c}81 \\
.8 \\
\end{array}$ & $\begin{array}{r}87 \\
.5 \\
\end{array}$ & 24 & RA & $\begin{array}{l}54 \\
.2 \\
\end{array}$ & $\begin{array}{l}86 \\
.4 \\
\end{array}$ & $\begin{array}{r}95 \\
.8 \\
\end{array}$ \\
\hline 7 & FRS & $\begin{array}{c}54 \\
.2 \\
\end{array}$ & $\begin{array}{c}90 \\
.9 \\
\end{array}$ & $\begin{array}{l}79 \\
.2\end{array}$ & 25 & CYS & $\begin{array}{r}25 \\
.0\end{array}$ & $\begin{array}{l}63 \\
.6 \\
\end{array}$ & $\begin{array}{l}50 \\
.0\end{array}$ \\
\hline 8 & $\begin{array}{c}\text { MDA } \\
\text { R }\end{array}$ & $\begin{array}{l}20 \\
.8\end{array}$ & $\begin{array}{l}63 \\
.6\end{array}$ & $\begin{array}{l}83 \\
.3\end{array}$ & 26 & UNH & $\begin{array}{l}37 \\
.5\end{array}$ & $\begin{array}{c}10 \\
0\end{array}$ & $\begin{array}{r}87 \\
.5\end{array}$ \\
\hline 9 & FA & $\begin{array}{l}54 \\
.2\end{array}$ & $\begin{array}{c}81 \\
.8\end{array}$ & $\begin{array}{c}10 \\
0\end{array}$ & 27 & LSP & $\begin{array}{l}45 \\
.8\end{array}$ & $\begin{array}{c}90 \\
.9\end{array}$ & $\begin{array}{r}79 \\
.2\end{array}$ \\
\hline 10 & $\begin{array}{c}\text { BSY } \\
\text { S }\end{array}$ & $\begin{array}{r}45 \\
.8 \\
\end{array}$ & $\begin{array}{l}68 \\
.2 \\
\end{array}$ & $\begin{array}{l}87 \\
.5 \\
\end{array}$ & 28 & AR & $\begin{array}{l}54 \\
.2 \\
\end{array}$ & $\begin{array}{l}81 \\
.8 \\
\end{array}$ & $\begin{array}{r}87 \\
.5 \\
\end{array}$ \\
\hline 11 & KTR & $\begin{array}{l}58 \\
.3\end{array}$ & $\begin{array}{c}90 \\
.9\end{array}$ & $\begin{array}{l}91 \\
.7\end{array}$ & 29 & DW & $\begin{array}{c}50 \\
.0\end{array}$ & $\begin{array}{l}81 \\
.8\end{array}$ & $\begin{array}{c}83 \\
.3\end{array}$ \\
\hline 12 & ZS & $\begin{array}{c}58 \\
.3\end{array}$ & $\begin{array}{c}81 \\
.8 \\
\end{array}$ & $\begin{array}{l}87 \\
.5\end{array}$ & 30 & RAJ & $\begin{array}{r}29 \\
.2\end{array}$ & $\begin{array}{l}63 \\
.6\end{array}$ & $\begin{array}{c}66 \\
.7\end{array}$ \\
\hline 13 & HW & $\begin{array}{c}50 \\
.0\end{array}$ & $\begin{array}{l}77 \\
.3\end{array}$ & $\begin{array}{l}95 \\
.8\end{array}$ & 31 & AWS & $\begin{array}{l}50 \\
.0\end{array}$ & $\begin{array}{l}77 \\
.3\end{array}$ & $\begin{array}{r}75 \\
.0\end{array}$ \\
\hline 14 & VAS & $\begin{array}{c}50 \\
.0\end{array}$ & $\begin{array}{c}68 \\
.2\end{array}$ & $\begin{array}{l}79 \\
.2\end{array}$ & 32 & $\begin{array}{c}\text { SNA } \\
\text { A }\end{array}$ & $\begin{array}{r}29 \\
.2\end{array}$ & $\begin{array}{l}81 \\
.8\end{array}$ & $\begin{array}{l}75 \\
.0\end{array}$ \\
\hline 15 & SPA & $\begin{array}{l}66 \\
.7\end{array}$ & $\begin{array}{r}86 \\
.4\end{array}$ & $\begin{array}{c}10 \\
0\end{array}$ & 33 & ARF & $\begin{array}{l}41 \\
.7\end{array}$ & $\begin{array}{l}63 \\
.6\end{array}$ & $\begin{array}{l}75 \\
.0\end{array}$ \\
\hline 16 & MM & $\begin{array}{r}37 \\
.5\end{array}$ & $\begin{array}{l}81 \\
.8\end{array}$ & $\begin{array}{c}58 \\
.3\end{array}$ & 34 & BEP & $\begin{array}{r}37 \\
.5\end{array}$ & $\begin{array}{l}77 \\
.3\end{array}$ & $\begin{array}{c}58 \\
.3\end{array}$ \\
\hline 17 & MFR & $\begin{array}{l}50 \\
.0 \\
\end{array}$ & $\begin{array}{r}63 \\
.6 \\
\end{array}$ & $\begin{array}{l}83 \\
.3 \\
\end{array}$ & 35 & FDN & $\begin{array}{l}54 \\
.2 \\
\end{array}$ & $\begin{array}{l}77 \\
.3 \\
\end{array}$ & $\begin{array}{r}83 \\
.3 \\
\end{array}$ \\
\hline 18 & RK & $\begin{array}{l}50 \\
.0 \\
\end{array}$ & $\begin{array}{l}77 \\
.3 \\
\end{array}$ & $\begin{array}{l}91 \\
.7 \\
\end{array}$ & & & & & \\
\hline
\end{tabular}

TABLE III. LEARNING RESULT IN THE HIGHEST (3), LOWER (2) AND THE LOWEST (1) CATEGORIES AT IMPLEMENTATION OF

\begin{tabular}{|c|c|c|c|c|c|c|c|c|c|}
\hline \multirow{2}{*}{$\begin{array}{c}- \\
\text { No } \\
\text {. }\end{array}$} & \multirow{2}{*}{$\begin{array}{c}\text { Stude } \\
\text { nt } \\
\text { Name }\end{array}$} & \multicolumn{3}{|c|}{$\begin{array}{c}\text { Learning } \\
\text { result at } \\
\text { implement } \\
\text { ation of }\end{array}$} & \multirow[t]{2}{*}{ No. } & \multirow{2}{*}{$\begin{array}{c}\text { Stude } \\
\text { nt } \\
\text { Name }\end{array}$} & \multicolumn{3}{|c|}{$\begin{array}{l}\text { Learning } \\
\text { result at } \\
\text { implement } \\
\text { ation of }\end{array}$} \\
\hline & & $\begin{array}{l}A \\
o \\
L\end{array}$ & $\begin{array}{l}A \\
f \\
L\end{array}$ & $\begin{array}{l}A \\
a \\
L\end{array}$ & & & $\begin{array}{l}A \\
o \\
L\end{array}$ & $\begin{array}{l}A \\
f \\
L\end{array}$ & $\begin{array}{l}A \\
a \\
L\end{array}$ \\
\hline 1 & $\begin{array}{c}\text { PIK } \\
\text { M }\end{array}$ & 2 & 2 & 2 & 19 & ST & 3 & 3 & 2 \\
\hline 2 & RTA & 2 & 2 & 2 & 20 & $\mathrm{~S}$ & 2 & 2 & 2 \\
\hline 3 & $\begin{array}{c}\text { MFS } \\
\mathrm{K}\end{array}$ & 2 & 2 & 2 & 21 & RKN & 2 & 3 & 2 \\
\hline 4 & MSR & 2 & 1 & 1 & 22 & AFN & 2 & 2 & 2 \\
\hline 5 & RED & 3 & 3 & 2 & 23 & EAP & 2 & 2 & 1 \\
\hline 6 & BBM & 2 & 2 & 2 & 24 & RA & 2 & 2 & 3 \\
\hline 7 & FRS & 2 & 3 & 2 & 25 & CYS & 1 & 1 & 1 \\
\hline 8 & $\begin{array}{c}\text { MDA } \\
\mathrm{R}\end{array}$ & 1 & 1 & 2 & 26 & UNH & 2 & 3 & 2 \\
\hline
\end{tabular}

\begin{tabular}{|c|c|c|c|c|c|c|c|c|c|}
\hline \multirow{2}{*}{$\begin{array}{c}- \\
\text { No } \\
\text {. }\end{array}$} & \multirow{2}{*}{$\begin{array}{c}\text { Stude } \\
\text { nt } \\
\text { Name }\end{array}$} & \multicolumn{3}{|c|}{$\begin{array}{c}\text { Learning } \\
\text { result at } \\
\text { implement } \\
\text { ation of }\end{array}$} & \multirow[t]{2}{*}{ No. } & \multirow{2}{*}{$\begin{array}{c}\text { Stude } \\
\text { nt } \\
\text { Name }\end{array}$} & \multicolumn{3}{|c|}{$\begin{array}{c}\text { Learning } \\
\text { result at } \\
\text { implement } \\
\text { ation of }\end{array}$} \\
\hline & & \begin{tabular}{l|}
$A$ \\
$o$ \\
$L$
\end{tabular} & \begin{tabular}{|l|}
$A$ \\
$f$ \\
$L$ \\
\end{tabular} & $\begin{array}{l}A \\
a \\
L\end{array}$ & & & $\begin{array}{l}A \\
o \\
L \\
\end{array}$ & \begin{tabular}{l|}
$A$ \\
$f$ \\
$L$ \\
\end{tabular} & $\begin{array}{c}A \\
a \\
L\end{array}$ \\
\hline 9 & FA & 2 & 2 & 3 & 27 & LSP & 2 & 3 & 2 \\
\hline 10 & $\begin{array}{c}\text { BSY } \\
\text { S }\end{array}$ & 2 & 2 & 2 & 28 & AR & 2 & 2 & 2 \\
\hline 11 & KTR & 2 & 3 & 2 & 29 & DW & 2 & 2 & 2 \\
\hline 12 & $\mathrm{ZS}$ & 2 & 2 & 2 & 30 & RAJ & 1 & 1 & 1 \\
\hline 13 & HW & 2 & 2 & 3 & 31 & AWS & 2 & 2 & 2 \\
\hline 14 & VAS & 2 & 2 & 2 & 32 & $\begin{array}{c}\text { SNA } \\
\text { A }\end{array}$ & 1 & 2 & 2 \\
\hline 15 & SPA & 3 & 2 & 3 & 33 & ARF & 2 & 1 & 2 \\
\hline 16 & MM & 2 & 2 & 1 & 34 & BEP & 2 & 2 & 1 \\
\hline 17 & MFR & 2 & 1 & 2 & 35 & FDN & 2 & 2 & 2 \\
\hline 18 & RK & 2 & 2 & 2 & & & & & \\
\hline
\end{tabular}

Table 4 show tabulation of data in Table 1 and 3 at implementation of AoL. Table 4 inform only students percept assessment as $\mathrm{AaL}$ that it attain the highest categories in learning result.

TABLE IV. TABULATION BETWEEN AMOUNT STUDENT THAT IT PERCEPT ASSESSMENT AS AOL, AFL AND AAL WITH AMOUNT STUDENT THAT IT REACH LEARNING RESULT OF THE HIGHEST, LOWER AND THE LOWEST CATEGORIES AT IMPLEMENTATION AOL

\begin{tabular}{|c|c|c|c|c|c|}
\hline Count & & & & \\
\hline & & \multicolumn{4}{|c|}{$\begin{array}{c}\text { Amount student that it } \\
\text { reach learning result of }\end{array}$} \\
\hline & & $\begin{array}{c}\text { The } \\
\text { lowest }\end{array}$ & $\begin{array}{c}\text { Ther } \\
\text { lower }\end{array}$ & $\begin{array}{c}\text { Thighest } \\
\text { Total }\end{array}$ \\
\hline \multirow{2}{*}{$\begin{array}{c}\text { Amount student } \\
\text { that it percept } \\
\text { assessment as }\end{array}$} & AoL & 1 & 2 & 0 & 3 \\
\cline { 2 - 6 } & AfL & 1 & 14 & 0 & 15 \\
\cline { 2 - 6 } & AaL & 2 & 12 & 3 & 17 \\
\hline \multicolumn{2}{|c|}{ Total } & 4 & 28 & 3 & 35 \\
\hline
\end{tabular}

Chi-Square Tests (Huck, 2008) is used to measure relationships between perception toward assessment with learning result at implementation AoL [9]. The result of measuring is shown in Table 5. Asymp. Sig. (2-sided) in Table 5 show the number that it is higher than 0,05 . It means, there is no relationships significantly between perception toward assessment with learning result at implementation AoL.

TABLE V. RESULT OF CHI-SQUARE TESTS BETWEEN PERCEPTION TOWARD ASSESSMENT WITH LEARNING RESULT AT IMPLEMENTATION AOL

\begin{tabular}{|c|c|c|c|}
\hline & Value & df & Asymp. Sig. (2-sided) \\
\hline Pearson Chi-Square & $5.324^{\mathrm{a}}$ & 4 & .256 \\
\hline Likelihood Ratio & 6.095 & 4 & .192 \\
\hline $\begin{array}{c}\text { Linear-by-Linear } \\
\text { Association }\end{array}$ & 1.951 & 1 & .162 \\
\hline
\end{tabular}




\begin{tabular}{|c|c|c|c|}
\hline & Value & df & Asymp. Sig. (2-sided) \\
\hline Pearson Chi-Square & $5.324^{\mathrm{a}}$ & 4 & .256 \\
\hline Likelihood Ratio & 6.095 & 4 & .192 \\
\hline $\begin{array}{c}\text { Linear-by-Linear } \\
\text { Association }\end{array}$ & 1.951 & 1 & .162 \\
\hline N of Valid Cases & 35 & & \\
\hline
\end{tabular}

TABLE VIII. TABULATION BETWEEN AMOUNT STUDENT THAT IT PERCEPT ASSESSMENT AS AOL, AFL AND AAL WITH AMOUNT STUDENT THAT IT REACH LEARNING RESULT OF THE HIGHEST, LOWER AND THE LOWEST CATEGORIES AT IMPLEMENTATION AAL

\begin{tabular}{|c|c|c|c|c|c|}
\hline \multicolumn{6}{|l|}{ Count } \\
\hline & & \multicolumn{3}{|c|}{$\begin{array}{c}\text { Amount student that it } \\
\text { reach learning result } \\
\text { categories of }\end{array}$} & \multirow[b]{2}{*}{ Tota } \\
\hline & & $\begin{array}{c}\text { The } \\
\text { lowest }\end{array}$ & lower & $\begin{array}{c}\text { The } \\
\text { highest }\end{array}$ & \\
\hline \multirow{3}{*}{$\begin{array}{l}\text { Amount student } \\
\text { that it percept } \\
\text { assessment as }\end{array}$} & AoL & 0 & 3 & 0 & 3 \\
\hline & AfL & 1 & 5 & 1 & 7 \\
\hline & $\mathrm{AaL}$ & 5 & 17 & 3 & 25 \\
\hline \multicolumn{2}{|l|}{ Total } & 6 & 25 & 4 & 35 \\
\hline
\end{tabular}

Chi-Square Tests is used to measure relationship between perception toward assessment with learning result at implementation AaL. The result of measuring is shown in Table 9. Asymp. Sig. (2-sided) in Table 9 show the number that it is higher than 0,05 . It means, there is no relationship significantly between perception toward assessment with learning result at implementation AaL.

TABLE IX. RESULT OF CHI-SQUARE TESTS BETWEEN PERCEPTION TOWARD ASSESSMENT WITH LEARNING RESULT AT IMPLEMENTATION AAL

\begin{tabular}{|c|c|c|c|}
\hline & Value & df & $\begin{array}{c}\text { Asymp. Sig. (2- } \\
\text { sided) }\end{array}$ \\
\hline Pearson Chi-Square & $1.451^{\mathrm{a}}$ & 4 & .835 \\
\hline Likelihood Ratio & 2.262 & 4 & .688 \\
\hline $\begin{array}{c}\text { Linear-by-Linear } \\
\text { Association }\end{array}$ & .134 & 1 & .714 \\
\hline N of Valid Cases & 35 & & \\
\hline
\end{tabular}

There is no relationships significantly between perception toward assessment with learning result at implementation AoL, AfL and AaL based on the Table 5, 7 and 9. However, if student's the highest and lower categories in learning result are added at implementation of AoL, AfL and AaL are always predominated by student which percept assessment successively as AaL, AfL and AoL. Amount of student which is obtaining the highest and lower categories in learning result at implementation of AoL is $15(42,9 \%), 14(40 \%)$ and $2(5,7 \%)$ student which percept assessment successively as AaL, AfL and AoL. Amount of student which is obtaining the highest and lower categories in learning result at implementation of AfL is $23(65,7 \%), 6(17,1 \%)$ and $0(0 \%)$ student which is percept assessment successively as AaL, AfL and AoL. Amount of student which is obtaining the highest and lower categories in learning result at implementation of AaL is $20(57,1 \%), 6(17,1 \%)$ and $3(8,6 \%)$ student which is percept assessment successively as AaL, AfL and AoL.

Generally, perception is the way in which something is regarded, understood or interpreted. Perception affect to someone behavior significantly [10]. 
Perception towards assessment is defined as student behavior recognize assessment in learning research moment conducted.

When student percept assessment as AoL, hence it's student delivery lecturer assessment activity because according to Earl (2003) that the teacher is key assessor at implementing AoL. Student is not thinking to involve in assessment activity because according to Funk (2009) that Student is not involving in assessment activity at implementation AoL.

Learning result of students increase when student percept assessment as AfL. Although the teacher is key assessor at implementing AfL, but the students realize involving themselves in assessment activity will increase their learning result. It is suitable statement Funk (2009) that the student is the best to increase it's learning. An example, student HW percepts assessment as AoL (at implementing AoL) reaches value 50 and his value becomes 95.8 when he percepts assessment as AfL at implementation AaL. Student MDAR percepts assessment as AoL (at implementing AoL) reaches value 20.8 and his value becomes 83.3 when he percepts assessment as AfL at implementation AaL.It is so happen student AFN. He percepts assessment as AfL (at implementing AfL) reaches value 68.2 and his value becomes 95.8 when he percepts assessment as $\mathrm{AaL}$ at implementation $\mathrm{AaL}$.

\section{CONCLUSION}

There is no relationships significantly between perception toward assessment with learning result at implementation AoL, AfL and AaL. However, if student's the highest and lower categories in result learning are added at implementation of AoL, AfL and AaL are always predominated by student which percept assessment successively as AaL, AfL and AoL. Amount of student which is obtaining the highest and lower categories in result learning at implementation of AoL are 15 (42,9\%), $14(40 \%)$ and $2(5,7 \%)$ student which percept assessment successively as AaL, AfL and AoL. Amount of student which is obtaining the highest and lower categories in result learning at implementation of AfL are $23(65,7 \%), 6$ $(17,1 \%)$ and $0(0 \%)$ student which is percept assessment successively as AaL, AfL and AoL. Amount of student which is obtaining the highest and lower categories in result learning at implementation of $\mathrm{AaL}$ are $20(57,1 \%)$, $6(17,1 \%)$ and $3(8,6 \%)$ student which is percept assessment successively as AaL, AfL and AoL.

\section{REFERENCES}

[1] E. a. Giles, "An-other Look at Assessment: Assessment in Learning," New Zealand Journal of Teachers'Work, vol. 8, no. 1, pp. 11-20, 2011.

[2] L. Earl, Assessmen as Learning: Using Classroom Assessment to Maximize Student Learning., Thousand Oaks, CA, : Corwin Press., 2003

[3] D. Wiliam, "Assessment: The Bridge between Teaching and Learning," Voice from the Middle, vol. 21, p. 2, 2013.

[4] U. a. Earl, "Summative and Formative: Confused by the Assessment Terms," New Zealand Journal of Teacher Work, vol. 7, no. 1, pp. 53-63, 2010 .
[5] K. a. G. D. Earl, "An-other Look at Assessment: Assessment in Learning," New Zealand Journal of Teachers'Work, vol. 8, no. 1, pp. 11-20, 2011

[6] T. Glasson, "Improving student achievement through Assessment for Learning," Curriculum \& Leadership Journal, vol. 6, no. 31, 2008.

[7] R. a. C. Stiggins, "What a difference a word makes: Assessmen FOR Learning rather than assessmen OF Learning helps students succeed," JSD, vol. 27, no. 1, 2006.

[8] A. Davies, K. Patzopoulos and K. and Gray, "Emphasising Assessment as Learning by Assessing Wiki Writing Assgnments Collaboratively and Publicly Online," Australasian Journal of educational Technology, vol. 27, no. 5, pp. 798-812, 2011.

[9] H. Alkharusi, " Canonical Correlational Models of Students' Perceptions of Assessment Task, Motivational Orientations, and Learning Strategies," International Journal of Instruction, vol. 6, no. 1, 2013.

[10] L. Cheng, Y. F. Wu and X. dan Liu, Students' perceptions of assessment task and Classroom Assessment environment. Languange Testing in Asia. Springer, Chinese: Chinese University , 2015.

[11] S. W. Huck, Reading Statistics and Research, New York: Harper and Row, 2008.

[12] I. Ajzen, "The Theory of Planned behavior," Organizational Behavior and Human Decision processes, vol. 50, no. 2, pp. 179$211,1991$. 